\title{
Energy based AODV Routing Algorithm with Sleep Mode in MANETs
}

\author{
Tripti Nema \\ Department of \\ M.Tech(CSE) Bist, \\ Bhopal
}

\author{
Akhilesh waoo \\ Department of \\ M.Tech(CSE) Bist, \\ Bhopal
}

\author{
P. S. Patheja \\ Department of \\ M.Tech(CSE) Bist, \\ Bhopal
}

\author{
Sanjay Sharma, Ph.D \\ Department of \\ M.Tech(CSE) MANIT, \\ Bhopal
}

\begin{abstract}
MANET became the focus of researchers as a promising technology for a broad range of applications due to their selforganizing, self-configuring capability in different mobile network scenarios. One significant area of research within ad hoc networks is energy consumption issue. The primary goal of ad hoc networks is to call for the energy-constrained protocols. Energy consumption evaluation methodology is introduced for the protocol consideration in different mobile network. AODV routing protocol performs well on assessment of energy consumption for the mobile ad hoc network with high node mobility. Nodes in MANET networks are basically battery operated, and thus have access to a limited amount of energy. This process proposes an Energy based Ad-Hoc on-Demand Routing algorithm that balances energy among nodes so that a minimum energy level is maintained among nodes and the lifetime of network is increased. This paper, focused on increasing the prolonged existence of node in the network. In this proposed work, one set the minimum energy threshold limit of a mobile node, when a node reach the minimum threshold limit the node goes to sleep mode, save energy and participate in the event as long as possible. The research papers are published to improve the network lifetime on the network layer. The construct of network scenarios and performance analysis is done on NS-2.34 to simulate both the AODV and AODVSleep under the similar scenario. This paper also compares and analyzes the simulation results with a popular on-demand routing protocol AODV to show the usefulness of this algorithm. From this simulation one finds that the overall MANET's efficiency is enhanced.
\end{abstract}

\section{Keywords}

Mobile Ad-Hoc Networks, AODV routing protocols, energy consumption, Sleep mode, mobility models, simulation analysis.

\section{INTRODUCTION}

Mobile Ad Hoc Networks (MANETs) represent the decentralized paradigms where clients themselves sustain the network in the absence of a central infrastructure. MANET does not operate under fixed topology means they are selforganising, self-administrating, self-healing type of network. In MANET, each mobile node operate as both a router and a terminal nodes which is a source or destination, thus the failure of some nodes operation can greatly hinder the performance of the network and also affect the basic accessibility of the network, i.e., energy exhaustion of nodes has been one of the main harm to the connectivity of MANET. Since the mobile nodes in MANET have limited battery power, so it is required to efficiently use energy of every node in MANET. MANET is a multi-hop, in which node can freely move in any direction and have limited battery power. In this type of networks, energy parameter plays an important role in the research. A reliable routing protocol for Mobile Ad hoc Networks (MANETs) keeps the energy consumption as low as possible. To estimate the energy consumption the numerous MANET routing protocols have been developed for network. The performance of DSDV [1] introduces large amounts of overhead to the network due to the requirement of the periodic update messages, and the overhead grows at the quantity of $O\left(N^{2}\right)$.DSR [2] is that it uses broadcast for route discovery while broadcast causes too much message forwarding traffic and energy consumption, especially when the network is large. AODV [3] AODV is based on both DSDV and DSR algorithm. It uses the route discovery and route maintenance practice of DSR. DSR packet carries the complete route information, while the packet of AODV only carries the destination address, it has less routing overhead than DSR. At the same time, AODV makes use of routing messages and sequence numbering. Here AODV is evaluated and analyzed from the aspect the energy utilization metric.

AODV protocol is a reactive routing protocol which finds route to destination when required. AODV consists of routing table which helps to differentiate between expiry and fresh routes. The routing table at node contains the sequence number and next hop information. The working of protocol is consists of two phases:

\section{Route discovery and}

2. Route maintenance.

In route discovery process, the source node generate RREQ packet, if the path to destination is not stored in the routing table, and pass it to the neighboring nodes. The neighboring nodes will pass it to their neighbor and so on. When the packet reached to the destination node, then destination node generates RREP (Route Reply) packet and send it back to the source node. Thus the path is established between source and destination node. In route maintenance process, the source node is being informed by RERR (Route Error) message in case of link breakage. Also the connectivity between the nodes is maintaining using Hello messages. There are two main factors that cause link failures are:

\section{Battery life time \\ II. Mobility}

Taking energy in consideration, there are three approaches that are usually used to achieve the energy-efficiency in MANETs [4]: Power-Control, Power-Save and MaximumLifetime routing. The Power-Control approach is allowing nodes to decide the least amount of transmission power level which is sufficient to maintain network connectivity and to pass the traffic with least energy, the objectives is to increase network capacity and sinking energy consumption. The Power-Save approach deals with the energy loss during the 
idle mode and this can be minimized by increasing the amount of time a node spends in the sleep mode. Lastly, the Maximum-Lifetime routing approach looks for the nodes that have minimum energy so that they can be avoided from the path.

The paper is organized as follows. Section II survey the related work to estimation of energy based Ad hoc routing protocols for MANET. Section III briefly describes the idea and mechanism of AODV routing protocols which improve the energy efficiency of MANET. Section IV introduces the design of the byte-based energy consumption formula. Section $\mathrm{V}$ makes the energy consumption assessment under our proposed mobility models. Section VI draws the conclusion of the paper.

\section{RELATED WORK}

Several routing algorithms use the link lifetime as well as the nodes' battery life time as routing metrics to allow the most reliable and energy efficient route to be selected for data transmission.

Signal Stability based Adaptive Routing has been proposed in [5], which the links are categorized into groups according to the signal strength metric. During the route discovery phase, each mobile node divides the connections between itself and its neighboring nodes into two groups, a strongly connected (SC) group and a weakly connected (WC) group.

Minimum Battery Cost Routing (MBCR) has been proposed in [6]. MBCR routing protocol calculates the sum of the residual power of all nodes in a path and uses it for selecting a path, but the method may choose a path in which there may exist mobile nodes with low power. Thus, these low power mobile nodes may cause path breakage.

Fei Dai et al [7] the basic idea of this paper was to save power by using efficient broadcasting techniques that are achieved to conduct broadcasting using directional antennas for ad hoc networks. This paper firstly focuses on energy consumption as well as forwarding packets directionally by introducing directional antennas. Syropoulos et al [8], have implemented the use of Directional Antennas for energy efficient communication in ad hoc networks.Jin-Man Kim et al., [9] introduced an Energy Mean Value algorithm to enhance AODV routing protocol and to improve the network lifetime of MANET.

Krishna Cheong Lau and Joseph H. Kang [10] the idea to increase energy efficiency, nodes in the network goes into a sleep mode and wake up at predetermined time slot(s) to snoop for transmissions from its instant neighbors. The knowledge of awakening slots for neighboring nodes is used to arrange the transmissions within the neighborhood. Finally, nodes adapt their sleeping cycles based on neighbor topology and remaining battery life in order to maximize the network lifetime also satisfying the latency requirements of sensor applications.

In [11] authors have proposed a protocol named RP AR which design was based on considering the substitution between energy efficiency and latency. The participating nodes required to uphold an information table related to its neighbors. Attainment and maintenance of such information requires significant exchange of information through beacon signals which involve lot of energy consumption hence energy efficiency is surrendered.
The protocol (ORTR) [12] has the limitation that the forwarding area of the nodes is not a shared carrier sensing area. That is why the nodes cannot listen to each other once one of them is broadcasting.

\section{PROPOSED WORK}

Finally, most of the research works in the literature have addressed the link lifetime and the energy information as routing metrics to improve the route selection mechanism of the routing protocol. To the best of our knowledge, this is the first work that introduces the link lifetime and the nodes' residual energy to enhance the route discovery process that allows the routes that satisfy the link lifetime and the energy requirements to be discovered.

Due to the transition time for powering on an element (wakeup time), there is a penalty to each off-period. The wake-up time is a time period during which power is consumed by the element but no service is delivered. The wake-up time defines the lower bound to the length of the off-period for an element. Due to the wake-up time, off periods should be kept as large as possible in order to reduce the fraction of time spent in wake-up. The upper bound to the off-periods is set by quality of service requirement. The power of a wireless node is very important factor due to limited energy sources our proposed work is based on the energy saving of a node. Each node in wireless network works as a router and participate in the routing mechanism, the energy of moving node are limited. When the energy of node lost it disappears from network it affects the routing mechanism of other node also result routing overhead for searching the alternate routes. The searching of alternate route can be made on the basis of the Residual energy of the neighboring node:

1. If the Energy of neighboring node is greater than 50 then update the cache of the node and then transfer the traffic through that node.

2. Otherwise, search for the node with maximum Residual Energy and update the cache then transfer the traffic through that node.

A proposed solution utilizes the node energy when it reaches the minimum energy level called minimum threshold. When the node reaches at minimum threshold 1 it goes to sleep mode

\section{SIMULATION MODEL}

To simulate the real moving behaviors of the nodes in a mobile ad hoc network we use NS-2. The evaluations are conducted with 50 nodes that are randomly scattered in a region of $800 \mathrm{~m} \mathrm{X} 600 \mathrm{~m}$ with 50 number of connection. These factors are taken as the critical scenario. Energy model includes the radio range of $250 \mathrm{~m}, 2 \mathrm{Mbps}$ of data rate. Initial energy supplied to each node is random. The power consumed during transmission and reception are $1.5 \mathrm{~W}$ and $1.0 \mathrm{~W}$ respectively. The traffic model used is CBR (Constant Bit Rate) with packet size of 512 bytes, rate 50 packets/s and simulation time of $100 \mathrm{~s}$. The simulation is done with the help of NS-2 [5] and traffic model is generated using engergy.tcl.

In this paper we concentrate on Constant Bit Rate (CBR) sources (i.e voice sources) and ftp sources (i.e file transfer). The source-destination pairs are chosen randomly over the network. This simulation evaluates the protocol using the following performance metrics.

A. Network lifetime

B. Packet delivery fraction 


\section{Variance of node remaining energy}

NS2 simulator is adopted in this paper to evaluate the performance of the proposed methodology and compare the traditional AODV based wireless ADHOC network with the proposed sleeping node methodology. The parameter values of simulation are as shown in table.

Table 1. Simulation Parameter

\begin{tabular}{|c|c|}
\hline Type & Values \\
\hline Channel & Channel/Wireless Channel \\
\hline $\begin{array}{c}\text { Radio Propagation } \\
\text { Model }\end{array}$ & Propagation/Tworayground \\
\hline Network Interface & Physical/Wirlessphy \\
\hline MAC & MAC/802_11 \\
\hline Interface Queue & Queue/Droptail/Priqueue \\
\hline Antenna & Antenna/Omniantenna \\
\hline Link Layer & LL \\
\hline $\begin{array}{c}\text { Interface Queue } \\
\text { Length }\end{array}$ & 50 \\
\hline Routing Protocol & AODV \\
\hline Simulation Time & $100 \mathrm{~s}$ \\
\hline
\end{tabular}

\section{RESULT}

The simulation results are as shown in table below. From the result summary one can draw a conclusion that the proposed methodology performs well compare to traditional AODV. The packet delivery rate has been increases and packet drop rate decreases. And also delay has been decreases using the proposed methodology. We have estimated (i) Energy consumption due to routing packets (ii) Routing overhead and (iii) Delivery ratio for Comparison between AODV and AODV -sleep protocols and following results was observed. Several simulations are performed using NS2 network simulator and using parameters shown in table 1. NS2 generates a name trace files analyzed using an AWK scripting. The performance study involves AODV routing protocol.

Table 2. Simulation Result Summary

\begin{tabular}{|c|c|c|}
\hline Parameter & Original Method & Proposed Method \\
\hline No. Of Nodes & 10 & 10 \\
\hline Packet Send & 11318 & 11357 \\
\hline Receive & 5732 & 9387 \\
\hline Routing Packets & 402 & 134 \\
\hline $\begin{array}{c}\text { End To End } \\
\text { Delay }\end{array}$ & 510.71 & 670.68 \\
\hline $\begin{array}{c}\text { No. Of Packet } \\
\text { Drop }\end{array}$ & 5458 & 1958 \\
\hline
\end{tabular}

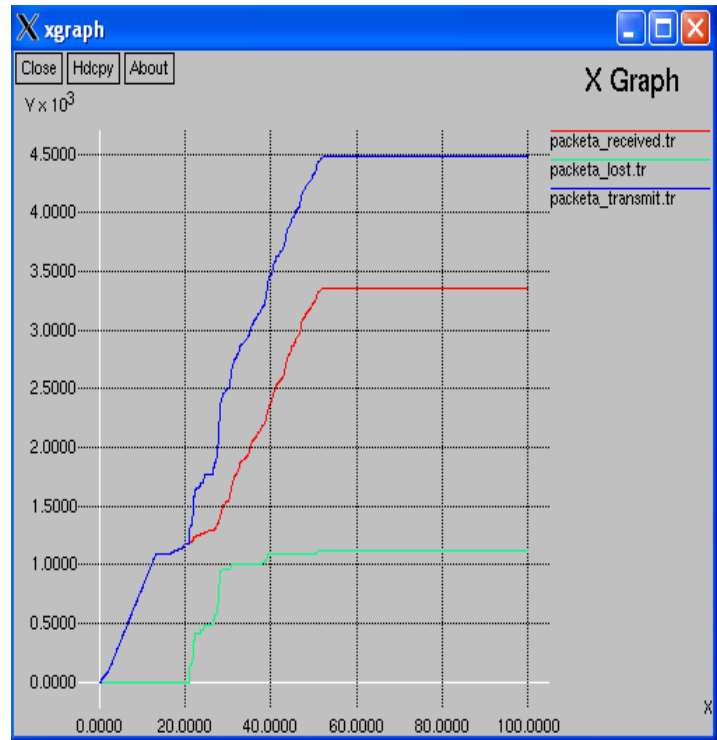

Fig. 1 Result summary of original algorithm

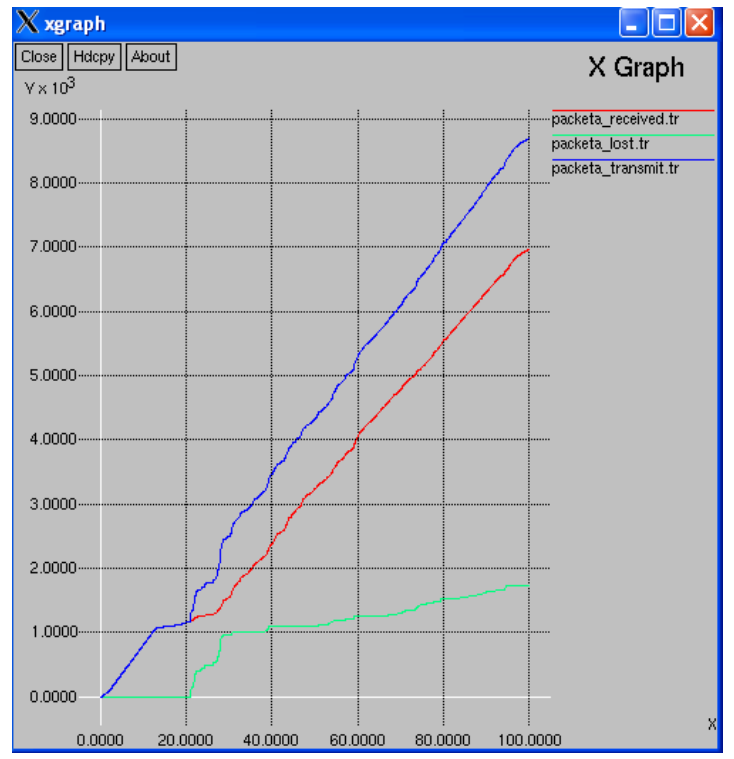

Fig. 2 Result summary of proposed algorithm

\section{CONCLUSION AND FUTURE WORK}

The above mention technique proposes a New-AODV protocol which enhances the network lifetime in an Ad-hoc network environment and simulated in NS2. Above all, each node's energy has a huge impact on the entire network lifetime. The proposed sleep mode scheme ensures significant improvement in power aware system. Hence, the effectiveness of sleep mode for the systems ultimately depends on the wake-up time for various nodes. The initiation of the awareness in the power management is proposed. In order to, overcome the energy based problem and prevent the link breakage. As a result, one can know that sleep mode to AODV protocol gives a transparent result to enhance the entire network lifetime. 


\section{REFERENCE}

[1] C. Perkins and P. Bhagwat, "Highly dynamic destination-sequenced distance-vector routing (DSDV) for mobile computers," in ACM SIGCOMM'94 Conference on Communications Architectures, Protocols and Applications, 1994, pp. 234-244.

[2] D.B. Johnson and D.A. Maltz, "Dynamic source routing in ad hoc wireless networks," in Mobile Computing, Imielinski and Korth, Eds. Kluwer Academic Publishers, 1996, vol. 353. pp. 153-181.

[3] C. Perkins, E. Belding-Royer and S. Das, "Ad hoc OnDemand Distance Vector (AODV) Routing," RFC 3561 (Experimental), Jul. 2003. [Online].

[4] J. Li, D. Cordes, and J. Zhang, "Power-aware routing protocols in ad hoc wireless networks," IEEE Trans. Wireless Commun., pp. 69-81, Dec. 2005.

[5] Dube, R., Rais, C.D., Wang, K.-Y., Tripathi, S.K., "Signal stability based adaptive routing (SSA) for ad hoc networks". IEEE Personal Communications 4, pp. 36-45, February 1997.

[6] Singh, S., Woo, M., Raghavendra, C.S., "Power-aware routing in mobile ad hoc networks". In: Proc. of $4^{\text {th }}$ Annual ACM/IEEE International Conference on Mobile Computing and Networking, pp. 181-190,1998.
[7] Fei Dai and Jie Wu, Efficient Broadcasting in Ad-Hoc Networks using Directional Antennas, Department of Computer Science and Engineering, Florida Atlantic University, Boca Raton, FL 33431.

[8] Kranakis, Danny Krizanc and Erric Williams Evangelos, Directional versus Omni Directional Antennas for Energy Consumption and k-Connectivity of Network of Sensors, October 15th 2004.

[9] Jin-Man Kim, Jong-Wook Jang, Kamrok Lee, "AODV Based Energy probability as compared to existing AODV routing protocol. Efficient Routing Protocol for Maximum Lifetime in MANET," in Proceedings of AICT/ICIVW IEEE, 2006

[10] Krishna Balachandran, Wing Cheong Lau and Joseph H. Kang, "Adaptive Sleeping and Awakening Protocol (ASAP) for Energy Efficient Adhoc Sensor Networks", 0-7803-8938-7/05/\$20.00 (C) 2005 IEEE.

[11] O. Chipara, Z. He, G. Xing, Q. Chen, X. Wang, C. Lu, J. Stankovic, and T. Abdelzaher. "Real-time power aware routing in sensor networks"Proc. 1WQoS'06, IEEE, pp.83-92, 2006.

[12] M. Zorzi and R. R. Rao. "Geographic random forwarding (GeRaF) for ad hoc and sensor networks: energy and latency performance," IEEE Trans. Mobile Computing, Yol.2, No.4, pp.34 